\title{
GeV electron beams from a centimetre-scale accelerator
}

\author{
W. P. LEEMANS ${ }^{1 \star \dagger}$, B. NAGLER ${ }^{1}$, A. J. GONSALVES ${ }^{2}$, Cs. TÓTH ${ }^{1}$, K. NAKAMURA ${ }^{1,3}$, C. G. R. GEDDES ${ }^{1}$, \\ E. ESAREY ${ }^{1 *}$, C. B. SCHROEDER ${ }^{1}$ AND S. M. HOOKER ${ }^{2}$
}

\author{
${ }^{1}$ Lawrence Berkeley National Laboratory, 1 Cyclotron Road, Berkeley, California 94720, USA \\ ${ }^{2}$ University of Oxford, Clarendon Laboratory, Parks Road, Oxford OX1 3PU, UK \\ ${ }^{3}$ Nuclear Professional School, University of Tokyo, 22-2 Shirane-shirakata, Tokai, Naka, Ibaraki 319-1188, Japan \\ *Also at: Physics Department, University of Nevada, Reno, Nevada 89557, USA \\ †e-mail:WPLeemans@|bl.gov
}

G igaelectron volt $(\mathrm{GeV})$ electron accelerators are essential to synchrotron radiation facilities and free-electron lasers, and as modules for high-energy particle physics. Radiofrequency-based accelerators are limited to relatively low accelerating fields $\left(10-50 \mathrm{MV} \mathrm{m}^{-1}\right)$, requiring tens to hundreds of metres to reach the multi-GeV beam energies needed to drive radiation sources, and many kilometres to generate particle energies of interest to high-energy physics. Laser-wakefield accelerators ${ }^{1,2}$ produce electric fields of the order $10-100 \mathrm{GV} \mathrm{m}^{-1}$ enabling compact devices. Previously, the required laser intensity was not maintained over the distance needed to reach $\mathrm{GeV}$ energies, and hence acceleration was limited to the $100 \mathrm{MeV}$ scale $^{3-5}$. Contrary to predictions that petawatt-class lasers would be needed to reach $\mathrm{GeV}$ energies ${ }^{6,7}$, here we demonstrate production of a high-quality electron beam with $1 \mathrm{GeV}$ energy by channelling a $40 \mathrm{TW}$ peak-power laser pulse in a $3.3-\mathrm{cm}-$ long gas-filled capillary discharge waveguide ${ }^{8,9}$.

Although it is straightforward to achieve acceleration gradients of $10-100 \mathrm{GV} \mathrm{m}^{-1}$ in laser-wakefield accelerators ${ }^{1,2}$, until recently the electron beams (e-beams) from such accelerators had energies $<200 \mathrm{MeV}$ with $100 \%$ energy spread $^{10}$. A breakthrough improvement in energy spread was obtained in 2004 by three groups $^{3-5}$ by interacting intense laser pulses with millimetre-scale gas jets to generate $70-200 \mathrm{MeV}$ beams with per-cent-level energy spread. For example, by using relatively large spot sizes, $r_{\mathrm{s}}=18 \mu \mathrm{m}$ $\left(1 / e^{2}\right.$ radius of the laser intensity profile), $170 \mathrm{MeV}$ e-beams were produced in 1-2-mm-long gas jets with the order of $0.5 \mathrm{nC}$ bunch charge using $30 \mathrm{fs}, 30 \mathrm{TW}$ laser pulses ${ }^{5}$. Using a 2-mm-long preformed plasma channel ${ }^{2}$ in a gas jet to guide the driving laser beam $^{4,11,12}$, enabled the production of $85 \mathrm{MeV}$ e-beams containing $0.3 \mathrm{nC}$ bunch charge, with only $9 \mathrm{TW}$ of laser peak power.

To scale laser-driven accelerators to $\mathrm{GeV}$ electron energies and beyond, two approaches had been proposed: (1) operate in initially uniform plasmas ${ }^{7,13}$ with petawatt (PW)-scale lasers and large laser spot sizes, or (2) channel guide the laser beam over centimetre-scale distances ${ }^{2,14,15}$. Without guiding (for example, without self-focusing or preformed channels), the laser-plasma interaction length is limited to the order of the Rayleigh range, $Z_{\mathrm{R}}$, (a few millimetres for $r_{\mathrm{s}}=25 \mu \mathrm{m}$ ). Relativistic self-guiding ${ }^{2,14}$ can extend the propagation distance of high-power pulses due to self-consistent modification of the plasma refractive index, but is limited by nonlinear effects such as the erosion of the leading edge of the laser pulse $\mathrm{e}^{16}$. Obtaining $\mathrm{GeV}$ energies without a channel therefore requires large laser spot sizes that increase $Z_{\mathrm{R}}$, but also increase the required laser power to PW levels ${ }^{6,7}$ and can result in an undesirable increase in the e-beam emittance.

A more efficient approach relies on channelling laser beams with smaller spot sizes over centimetre-scale distances. Theory and simulation indicate that such channel-guided accelerators could produce $\mathrm{GeV}$ e-beams with 10-50 TW of laser power ${ }^{2,4,14}$. However, simply making the accelerator longer is not sufficient. Phase slippage occurs between relativistic particles and the wake, because the wake has a phase velocity less than the vacuum speed of light. The linear dephasing length, $L_{\mathrm{d}}=\lambda_{\mathrm{p}}^{3} / \lambda^{2} \propto n_{\mathrm{p}}^{-3 / 2}$, over which electrons outrun the wake and slip into the decelerating phase, limits the distance over which acceleration occurs. Here $\lambda_{\mathrm{p}}$ is the plasma wavelength, $\lambda$ is the laser wavelength and $n_{\mathrm{p}}$ is the plasma density. For laser intensities $I \leq 10^{18} \mathrm{~W} \mathrm{~cm}^{-2}$, a rough estimate of the electron energy gain over a distance $L_{\mathrm{d}}$ in a channel-guided laser-wakefield accelerator ${ }^{2,14}$ can be obtained from $W(\mathrm{GeV}) \sim 0.4 I\left(\mathrm{Wcm}^{-2}\right) / n_{\mathrm{p}}\left(\mathrm{cm}^{-3}\right) \sim 0.9\left(\lambda_{\mathrm{p}} / r_{\mathrm{s}}\right)^{2} P(\mathrm{TW})$; where $P$ is the laser peak power (in TW). In the 2004 experiments, matching acceleration length to $L_{\mathrm{d}}$ led to the production of lowenergy-spread e-beams ${ }^{3-5}$.

Previously we created plasma channels ${ }^{17}$ in a gas jet with the 'ignitor-heater' technique, where a plasma column is ionized and then heated by two precursor laser pulses ${ }^{4,11,18}$. Owing to laser heating being inefficient at low densities, suitable plasma channels could only be produced at densities $>10^{19} \mathrm{~cm}^{-3}$, limiting $L_{\mathrm{d}}$ and restricting e-beam energies to about $100 \mathrm{MeV}$.

We overcame the limitations of gas-jet experiments by using a gas-filled capillary discharge waveguide ${ }^{8,9}$ to guide relativistically intense laser pulses in centimetre-scale, lower density plasma channels. The experiments used a $10 \mathrm{~Hz}$ repetition rate Ti:sapphire 


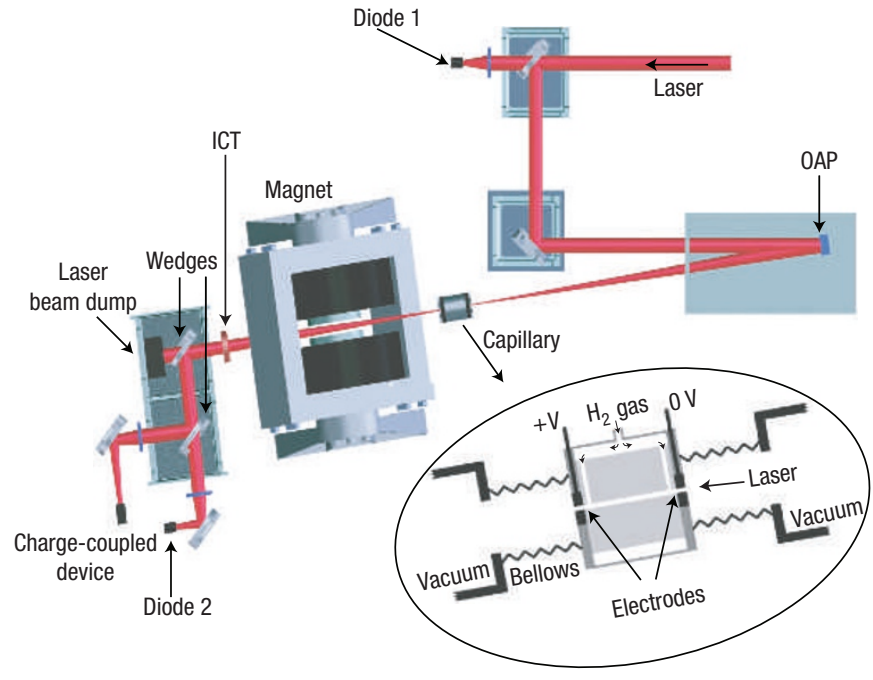

Figure 1 Schematic diagram of the capillary-guided laser-wakefield accelerator. The plasma channel was formed in a hydrogen-filled capillary discharge waveguide (see inset). Hydrogen gas was introduced into the capillary waveguide using two gas slots in the $225 \mu \mathrm{m}$ capillary and three in the $310 \mu \mathrm{m}$ capillary. A discharge was struck between two electrodes located at each end of the waveguide, using a high-voltage pulser. The pulser used a $2.7 \mathrm{nF}$ capacitor charged to $20 \mathrm{kV}$. The laser beam was focused onto the entrance of the capillary using an $f / 25$ off-axis parabola (OAP). The guiding efficiency was measured using a pair of optical diodes (diode 1 and 2) that monitored the amount of laser energy at the entrance and exit of the capillary. The laser beam exiting the capillary was monitored on a 12-bit charge-coupled device camera ( $20 \mu \mathrm{m}$ resolution), after having been attenuated with a pair of reflective wedges and optical attenuators. The e-beam was analysed using an integrating current transformer (ICT) and a 1.2 T broadband magnetic spectrometer (energy range of $0.03-0.15$ and $0.175-1.1 \mathrm{GeV}$ in a single shot). The e-beam was deflected downwards (into the page) and detected using phosphor screens imaged onto four synchronously triggered charge-coupled device cameras (not shown).

laser system $(\lambda=810 \mathrm{~nm})$ delivering pulses as short as $40 \mathrm{fs}$ full-width at half-maximum with up to 40 TW peak power (Fig. 1). These pulses were focused by a $2 \mathrm{~m}$ focal length offaxis parabola $(f / 25)$ to $r_{\mathrm{s}}=25 \mu \mathrm{m}$ at the capillary entrance. The capillaries ${ }^{8}$ were laser-machined into 33-mm-long sapphire blocks with diameters ranging from $190 \mu \mathrm{m}$ to $310 \mu \mathrm{m}$. Hydrogen gas, introduced through holes near the capillary ends, was ionized by striking a discharge between electrodes at the capillary ends. Measurements ${ }^{8}$ and modelling ${ }^{19,20}$ showed that a fully ionized, approximately parabolic channel is formed. Previous experiments ${ }^{9}$ demonstrated channelling of non-relativistically intense laser pulses with $I \lesssim 10^{17} \mathrm{~W} \mathrm{~cm}^{-2}$ in $30-50$-mm-long capillaries, which did not generate e-beams.

Guiding was optimized by adjusting the initial gas density and the delay between onset of the discharge current and arrival of the laser pulse (see Fig. 2a). Channel transmission correlated with discharge current (Fig. 2a) and, for low power ( $<5 \mathrm{TW}$ ), transmission was above $90 \%$ for densities ranging from 1.0 to $4.0 \times 10^{18} \mathrm{~cm}^{-3}$ in $\mathrm{a} \sim 100 \mathrm{~ns}$ timing window. Figure $2 \mathrm{~b}, \mathrm{c}$ shows laser beam profiles at the waveguide entrance and exit for $40 \mathrm{TW}$ laser pulses with an input intensity $\sim 10^{18} \mathrm{~W} \mathrm{~cm}^{-2}$ and a plasma density of $\simeq 2.7 \times 10^{18} \mathrm{~cm}^{-3}$. This intensity is sufficiently high for large-amplitude wake generation, self-trapping and high-gradient electron acceleration as observed in the experiment (see below). The guiding performance was highly sensitive to input-beam
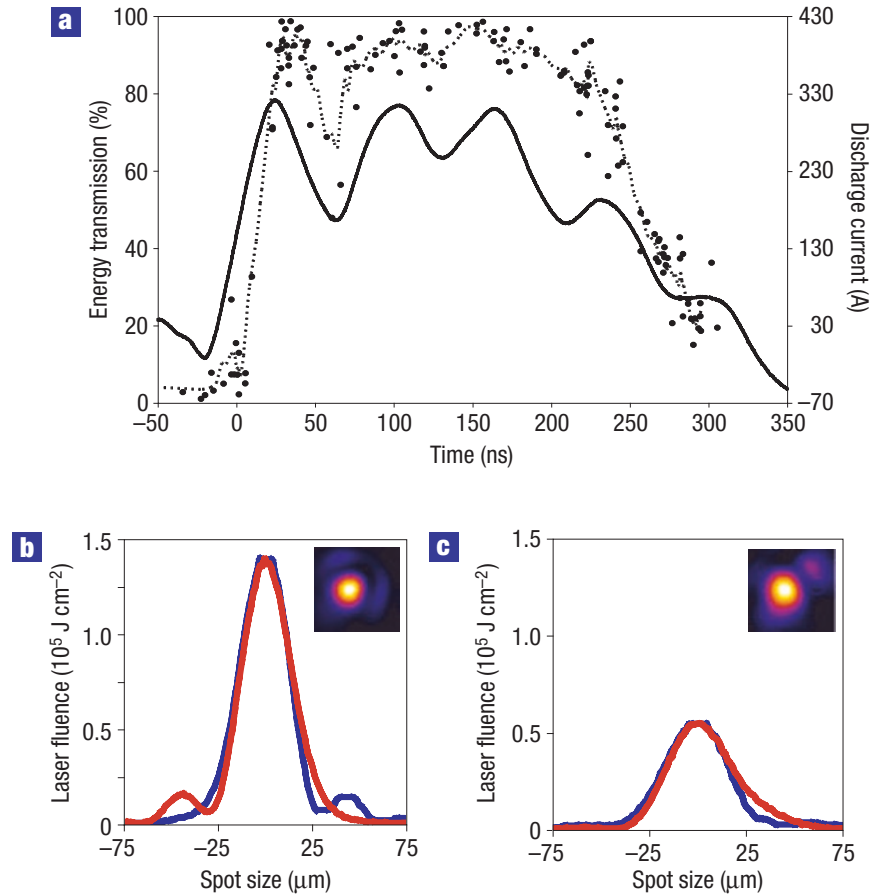

Figure 2 Capillary transmission at 5 TW peak input power versus time of arrival of the laser pulse after the onset of the discharge, and mode profiles of the input and output laser beam at $\mathbf{4 0} \mathrm{TW}$ peak power. a, The discharge current (solid line, right axis) and laser pulse energy transmission (circles, left axis) versus arrival time of the laser at the capillary for laser pulses $<5 \mathrm{TW}$. The dotted curve is the average transmission. b,c, The transverse spatial profiles of laser pulses with an input peak power of $40 \mathrm{TW}$ at the entrance (b) and exit (c) of the 3.3-cm-long gas-filled capillary discharge waveguide (diameter $=190 \mu \mathrm{m}$ ). The blue (red) curve is the horizontal (vertical) lineout. The horizontal and vertical spot sizes at the entrance were $r_{\mathrm{s} x}=25 \mu \mathrm{m}$ and $r_{\mathrm{s} y}=27 \mu \mathrm{m}$, respectively, and $r_{\mathrm{sx}}=31 \mu \mathrm{m}$ and $r_{s y}=34 \mu \mathrm{m}$ at the exit. The plasma density was $\simeq 3.2 \times 10^{18} \mathrm{~cm}^{-3}$. The $20 \%$ increase in spot size at the exit may be caused by imperfect mode matching. The energy transmission at this laser power was about $65 \%$. Combined with the increase in laser spot size this results in a decrease in laser peak fluence from $1.3 \times 10^{5} \mathrm{~J} \mathrm{~cm}^{-2}$ to $0.5 \times 10^{5} \mathrm{~J} \mathrm{~cm}^{-2}$. Assuming that the laser pulse duration remains constant between the entrance and exit of the capillary, the peak intensity of the laser was reduced from 3 to $1.2 \times 10^{18} \mathrm{~W} \mathrm{~cm}^{-2}$.

alignment, with $15 \mu \mathrm{m}$ displacement away from the optimum location (based on the quality of the guided beam) resulting in transmission drops of the order of $20 \%$. Note that without a preformed plasma channel (laser injected ahead of discharge), transmission was below $5 \%$ and bulk damage was sustained to the capillary channel walls, indicating that self-ionization and relativistic self-focusing could not be relied on for guiding, as expected from short-pulse propagation theory ${ }^{16}$.

Electron bunch energy was measured by a $1.2 \mathrm{~T}$ singleshot magnetic spectrometer that deflected the electrons vertically downwards onto a 1.2-m-long phosphor screen, covering energies from $0.03 \mathrm{GeV}$ to $1.1 \mathrm{GeV}$. E-beam divergence and energy spread were calculated from the data assuming a symmetric e-beam profile, and by using the imaging properties of the spectrometer, obtained from magnetic field maps and a secondorder electron transport model ${ }^{21}$. Divergence was determined from the e-beam size in the horizontal plane, taking into account the transverse defocusing properties of the magnet. Energy spread was calculated by deconvolving the effect of finite divergence 

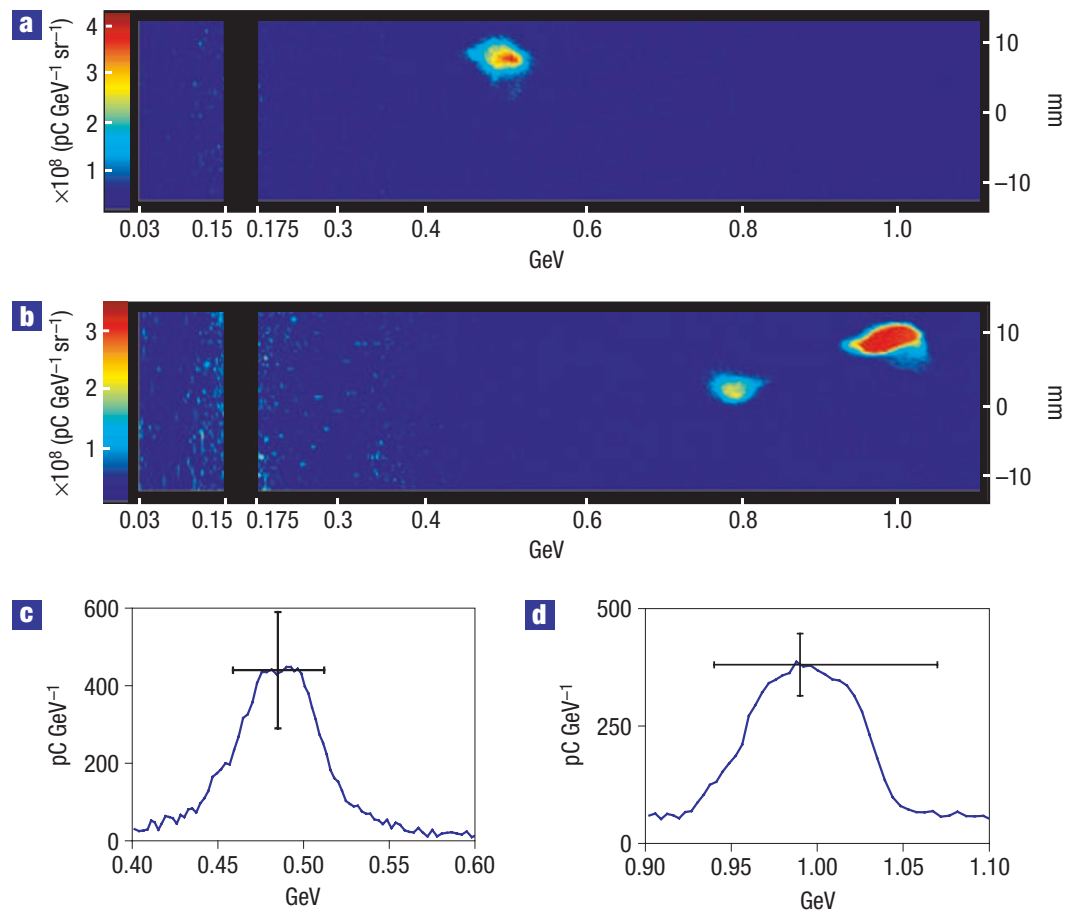

Figure 3 Single-shot e-beam spectra of the capillary-guided accelerator. a,b, Examples of bunches at $0.50_{-0.015}^{+0.02} \mathrm{GeV}(5.6 \%$ r.m.s. energy spread, $2.0 \mathrm{mrad}$ divergence r.m.s., $\sim 50 \mathrm{pC}$ charge) (a) and $1.0_{-0.05}^{+0.08} \mathrm{GeV}(2.5 \%$ r.m.s. energy spread, $1.6 \mathrm{mrad}$ divergence r.m.s., $\sim 30 \mathrm{pC})$ (b). The horizontal axis is the beam energy and the vertical axis is the beam size in the undeflected (horizontal) plane. The colour scale denotes the bunch charge in pC $\mathrm{GeV}^{-1} \mathrm{sr}^{-1}$. The $0.5 \mathrm{GeV}(1.0 \mathrm{GeV})$ beam shown was obtained in the $225(310) \mu \mathrm{m}$ capillary with a density of $\simeq 3.5 \times 10^{18}\left(4.3 \times 10^{18}\right) \mathrm{cm}^{-3}$ and input laser power of $12 \mathrm{TW}(40 \mathrm{TW})$. The black stripe denotes the energy range not measured by the spectrometer. $\ln \mathbf{b}$, a second beam at $0.8 \mathrm{GeV}$ is also visible. Note that the energy spread and divergence are obtained after including the imaging properties of the spectrometer. The energy spread at $1 \mathrm{GeV}$ may actually be less as the energy resolution is limited to $2.4 \%$ at $1 \mathrm{GeV}$ and there is slight saturation of the image. c,d, Vertically integrated spectra for the 0.5 (c) and $1.0 \mathrm{GeV}$ (d) beams. The vertical axis is the charge density in $\mathrm{pC} \mathrm{GeV}^{-1}$. The vertical error bar arises from uncertainty in calibration of the phosphor screen as a charge monitor $( \pm 17 \%)$. The horizontal error bar is due to the uncertainty in entrance angle of the e-beam resulting in an uncertainty in its energy. The spectrometer did not use an input slit, but the angular acceptance was limited by the transport beam pipe. For the $0.5 \mathrm{GeV}(1 \mathrm{GeV})$ beam, this gives an uncertainty in central energy of $+2 \%,-1.5 \%(+8 \%,-5 \%)$. In addition, for the $0.5 \mathrm{GeV}$ beam, sufficient statistics were obtained to include the shot-to-shot fluctuation, which amounted to $\pm 5 \%$ in mean energy and $\pm 30 \%$ in charge. Hence, the convolution of those factors are shown in $\mathbf{c}$, which are $+5.4 \%,-5.2 \%$ in mean energy and $\pm 34 \%$ in charge. The fluctuation in central energy was correlated with fluctuations in laser power.

from the measured e-beam profile. Charge was obtained from the phosphor screen, which was cross-calibrated against an integrating current transformer.

Figure 3 shows energy spectra of (a) $0.5 \mathrm{GeV}$ and (b) $1.0 \mathrm{GeV}$ beams, obtained with 12 TW ( 73 fs input) and 40 TW ( $38 \mathrm{fs}$ input) laser pulses, respectively. In both cases the e-beams had per-centlevel energy spread and a divergence of 1.2-2.0 mrad (r.m.s.).

Beams at $\sim 0.5 \mathrm{GeV}$ were obtained using a $225-\mu \mathrm{m}$-diameter capillary for a density of $\simeq 3.2$ to $3.8 \times 10^{18} \mathrm{~cm}^{-3}$ and for laser power ranging from as low as $12 \mathrm{TW}$ (using $73 \mathrm{fs}$ ) to $18 \mathrm{TW}$ (using $40 \mathrm{fs}$ ). The laser pulse energy transmission was observed to decrease from near $100 \%$ for input powers below $5 \mathrm{TW}$ to less than $70 \%$ for input powers above $18 \mathrm{TW}$, consistent with laser energy transfer to the wake and e-beams.

The performance of the $225-\mu \mathrm{m}$-diameter capillary-guided accelerator was found to be reproducible for delays between the laser arrival and onset of the discharge of $80-110 \mathrm{~ns}$ (that is, a 30 ns timing window) and 12 TW laser peak power. Every laser shot resulted in an e-beam at $0.48 \mathrm{GeV} \pm 6 \%$ and an r.m.s. spread $<5 \%$. Fluctuations in e-beam energy were directly correlated with those in laser power. For lower power $(<12 \mathrm{TW})$ no e-beams were observed, suggesting that the wake amplitude was below the self-trapping threshold. For higher power ( $>12 \mathrm{TW})$, the e-beam spectra typically showed significant structure (larger spread and multiple spots) and had much larger divergence, consistent with the wakefields exhibiting strong transverse structure in these relatively narrow channels, with a correspondingly strong impact on trapping (transverse wavebreaking) and focusing of the beams. In addition, the e-beam energy was lower and the bunch charge higher, suggesting that at these higher power levels more particles are trapped and that trapping occurs sooner in the channel, resulting in significant beam loading and reduction of the wakefield as well as improper matching of the acceleration length to $L_{\mathrm{d}}$.

The GeV e-beam was obtained in a 310- $\mu$ m-diameter channel capillary for $P=40$ TW and a density $\simeq 4.3 \times 10^{18} \mathrm{~cm}^{-3}$. In this larger diameter channel, transverse wakefields are reduced but the guiding properties are less ideal as this capillary requires a larger input spot size for matching than was used in the experiments. For lower laser power $(<38 \mathrm{TW})$, no e-beams were observed. For higher laser powers, the spectrum always showed structure with significant shot-to-shot fluctuations due in part to the self-trapping mechanism being sensitive to small variations in the laser and plasma parameters ${ }^{11}$. The dynamics of trapping, dephasing, beam loading ${ }^{11,22}$ and hosing ${ }^{23}$ may be responsible for the second spatially displaced bunch observed near $0.8 \mathrm{GeV}$ in Fig. 3b. Such features are observed in numerical simulations, owing to trapping of a second 
electron bunch in a wake bucket behind the first ${ }^{11,22}$. These issues as well as the importance of matching $L_{\mathrm{d}}$ in these structures are being further explored.

Although the performance of the accelerator at $1 \mathrm{GeV}(310-\mu \mathrm{m}$ diameter capillary) was less stable than at $0.5 \mathrm{GeV}(225-\mu \mathrm{m}-$ diameter capillary), it demonstrates the possibility of compact $\mathrm{GeV}$ accelerators driven by only a few tens of TW of laser power. To ensure stability may require some means of controlled particle injection via laser triggering ${ }^{24,25}$.

For $r_{\mathrm{s}}=25 \mu \mathrm{m}$, the simple scaling law discussed above predicts $\sim 0.27 \mathrm{GeV}$ using $18 \mathrm{TW}$ and $n_{\mathrm{p}}=3 \times 10^{18} \mathrm{~cm}^{-3}$; and $\sim 0.5 \mathrm{GeV}$ using $40 \mathrm{TW}$ and $n_{\mathrm{p}}=4 \times 10^{18} \mathrm{~cm}^{-3}$. These values are about a factor of two lower than the measured maximum electron energy (Fig. 3). Contrary to the experiment, however, the simple scaling laws do not include the full channel properties, nor effects of laser pulse evolution, self-focusing and e-beam loading on the plasma wake. For example, simulations indicate that pulse evolution is essential to the trapping and acceleration process. As the pulse propagates, it self-modulates and steepens such that the plasma electrons are completely blown out from the region of the axis (cavitation or bubble regime ${ }^{13}$ ), at which point electrons are selftrapped and accelerated from the background plasma in a manner similar to that described in previous experiments on high-quality beam production at the $100 \mathrm{MeV}$ level ${ }^{4,11}$.

The GeV-class electron beams from these centimetre-scale structures offer unique applications. The short wavelength of the plasma accelerating structure results in femtosecondduration bunches ${ }^{26}$ ( $>10 \mathrm{kA}$ peak current), well suited for driving pulsed radiation sources. These devices may allow compact femtosecond free-electron lasers producing $\mathrm{keV} \mathrm{X}$-rays using existing centimetre-scale period undulators and, in general, provide intrinsically synchronized sources of femtosecond pulses of electrons and radiation tunable from $\mathrm{X}$-ray ${ }^{27}$ to $\mathrm{THz}^{28}$ frequencies. Furthermore, it is anticipated that longer accelerating structures can be made by staging capillary discharge waveguides, thereby opening a path to compact accelerators beyond the multi-GeV level for high-energy physics applications.

Received 3 July 2006; accepted 29 August 2006; published 24 September 2006.

\section{References}

1. Tajima, T. \& Dawson, J. M. Laser electron accelerator. Phys. Rev. Lett. 43, 267-270 (1979).

2. Esarey, E., Sprangle, P., Krall, J. \& Ting, A. Overview of plasma-based accelerator concepts. IEEE Trans. Plasma Sci. 24, 252-288 (1996).
3. Mangles, S. P. D. et al. Monoenergetic beams of relativistic electrons from intense laser-plasma interactions. Nature 431, 535-538 (2004).

4. Geddes, C. G. R. et al. High-quality electron beams from a laser wakefield accelerator using plasma-channel guiding. Nature 431, 538-541 (2004).

5. Faure, J. et al. A laser-plasma accelerator producing monoenergetic electron beams. Nature 431, 541-544 (2004).

6. Lifschitz, A. F., Faure, J., Malka, V. \& Mora, P. GeV wakefield acceleration of low energy electron bunches using petawatt lasers. Phys. Plasmas 12, 093104 (2005).

7. Gordienko, S. \& Pukhov, A. Scalings for ultrarelativistic laser plasmas and quasimonoenergetic electrons. Phys. Plasmas 12, 043109 (2005)

8. Spence, D. J. \& Hooker, S. M. Investigation of a hydrogen plasma waveguide. Phys. Rev. E 63, 015401 (2001)

9. Butler, A., Spence, D. J. \& Hooker, S. M. Guiding of high-intensity laser pulses with a hydrogen-filled capillary discharge waveguide. Phys. Rev. Lett. 89, 185003 (2002).

10. Malka, V. et al. Electron acceleration by a wake field forced by an intense ultrashort laser pulse. Science 298, 1596-1600 (2002).

11. Geddes, C. G. R. et al. Production of high-quality electron bunches by dephasing and beam loading in channeled and unchanneled laser plasma accelerators. Phys. Plasmas 12, 056709 (2005).

12. Geddes, C. G. R. et al. Guiding of relativistic laser pulses by preformed plasma channels. Phys. Rev. Lett. 95, 145002 (2005).

13. Pukhov, A. \& Meyer-ter-Vehn, J. Laser wake field acceleration: the highly non-linear broken-wave regime. Appl. Phys. B 74, 355-361 (2002).

14. Leemans, W. P. et al. Plasma guiding and wakefield generation for second-generation experiments. IEEE Trans. Plasma Sci. 24, 331-342 (1996).

15. Hubbard, R. F. et al. Trapping and acceleration of nonideal injected electron bunches in laser wakefield accelerators. IEEE Trans. Plasma Sci. 33, 712-722 (2005).

16. Sprangle, P., Esarey, E. \& Ting, A. Nonlinear theory of intense laser-plasma interactions. Phys. Rev. Lett. 64, 2011-2014 (1990).

17. Durfee, C. G. III \& Milchberg, H. M. Light pipe for high intensity laser pulses. Phys. Rev. Lett. 71, 2409-2411 (1993).

18. Volfbeyn, P., Esarey, E. \& Leemans, W. Guiding of laser pulses in plasma channels created by the ignitor-heater technique. Phys. Plasmas 6, 2269-2277 (1999).

19. Bobrova, N. A. et al. Simulations of a hydrogen-filled capillary discharge waveguide. Phys. Rev. E 65, 016407 (2002).

20. Broks, B. H. P., Garloff, K. \& van der Mullen, J. J. A. M. Nonlocal-thermal-equilibrium model of a pulsed capillary discharge waveguide. Phys. Rev. E 71, 016401 (2005).

21. Makino, K. \& Berz, M. Cosy infinity version 8. Nucl. Instrum. Methods Phys. Res. A 427, 338 (1999).

22. Tsung, F. S. et al. Near-GeV-energy laser-wakefield acceleration of self-injected electrons in a centimeter-scale plasma channel. Phys. Rev. Lett. 93, 185002 (2004).

23. Deng, S. et al. Hose instability and wake generation by an intense electron beam in a self-ionized gas. Phys. Rev. Lett. 96, 045001 (2006).

24. Umstadter, D., Kim, J. K. \& Dodd, E. Laser injection of ultrashort electron pulses into wakefield plasma waves. Phys. Rev. Lett. 76, 2073-2076 (1996).

25. Esarey, E., Hubbard, R. F., Leemans, W. P., Ting, A. \& Sprangle, P. Electron injection into plasma wake fields by colliding laser pulses. Phys. Rev. Lett. 79, 2682-2685 (1997).

26. van Tilborg, J. et al. Temporal characterization of femtosecond laser-plasma-accelerated electron bunches using terahertz radiation. Phys. Rev. Lett. 96, 014801 (2006).

27. Catravas, P., Esarey, E. \& Leemans, W. P. Femtosecond x-rays from Thomson scattering using laser wakefield accelerators. Meas. Sci. Technol. 12, 1828-1834 (2001).

28. Leemans, W. P. et al. Observation of THz emission from a laser-plasma accelerated electron bunch crossing a plasma-vacuum boundary. Phys. Rev. Lett. 91, 074802 (2003).

\section{Acknowledgements}

This work was supported by the US Department of Energy and the Engineering and Physical Sciences Research Council, UK. We greatly appreciate contributions from C. Filip, E. Michel, B. Shadwick, M. Dickinson, D. Syversrud, J. Wallig, N. Ybarrolaza, M. Witney, T. Rowlands-Rees and D. Jaroszynski. Correspondence and requests for materials should be addressed to W.P.L.

\section{Competing financial interests}

The authors declare that they have no competing financial interests.

Reprints and permission information is available online at http://npg.nature.com/reprintsandpermissions/ 